\title{
Open Reduction without Fusion of Bilateral Jumped Lumbosacral Facet Dislocation Associated with Symphysis Pubis Separation and Cauda Equina Syndrome : Case Report
}

\author{
Sang-Bong Ko, Sang-Wook Lee \\ Department of Orthopaedic Surgery, Catholic University of Daegu School of Medicine, Daegu, Korea
}

\begin{abstract}
This paper reports a rare case of a lumbosacral dislocation associated with symphysis pubis separation and cauda equina syndrome. A 39-year-old male who diagnosed traumatic lumbosacral dislocation underwent an open reduction without fusion. After an open reduction and internal fixation of a symphysis pubis separation, a missed lumbosacral dislocation was diagnosed and an open reduction was performed without fusion. Due to the symphysis pubis separation, the patient was not allowed weight bearing for 3 months, which then began from wheel chair ambulation. At the 6-month follow up evaluation, there was no back pain but the patient reported mild S1 nerve root sensory symptoms. Lumbosacral dislocation is common in high energy polytrauma patients and can be misdiagnosed. However, prompt reduction without fusion is a good alternative treatment.
\end{abstract}

Key Words: Lumbosacral facet dislocation, Polyradiculopathy, Open reduction, Non-fusion

\section{Introduction}

Lumbosacral jumped facet are rare injuries caused by high-energy trauma with a rotational component in the setting of hyperflexion. This injury is encountered most often in poly-trauma patients, and can be missed in many cases. Most patients suffer from minor or no neurological injury. The typical presentation includes L5 slippage over the sacrum, a less frequent fracture line crossing the sacrum, multiple lumbar transverse process fractures and ipsilateral renal contusion. Fewer than 21 cases of fracture-dislocations of the fifth lumbar vertebra on the sacrum have been reported.

Most papers published have been case reports [1]. The optimal treatment of these rare high-energy lesions is con- troversial but an open reduction and fusion with instrumentation were performed in almost all of the 12 papers reported. The authors report a case of a lumbosacral dislocation associated with symphysis pubis separation, secondary cauda equine syndrome after fixation of symphysis pubis separation, and the surgical reduction without fusion or instrumentation.

\section{Case Report}

A 39-year-old male was injured after being trapped by his own van parked on a sidewalk and falling after being pushed by a taxi bumping against the side of the van twice. He was extricated from under the car by a 119 rescuer. Upon arrival at the emergency room, he showed no loss of

Received Sep 28, 2009; 1st Revised Dec 30, 2009; 2nd Revised Jan 22, 2010; 3rd Revised Jan 31, 2010; Accepted Jan 31, 2010

Corresponding author: Sang-Bong Ko, MD

Department of Orthopaedic Surgery, Daegu Catholic University Medical Center,

Daemyeong 4-dong, Nam-gu, Daegu 705-718, Korea

Tel: +82-53-650-4283, Fax: +82-53-626-4272, E-mail: bong@ cu.ac.kr 
consciousness. An examination demonstrated multiple deep abrasions over the entire body and extremities. A physical examination of the pelvis revealed tenderness on the symphysis pubis area. There were no neurological deficits except for a tingling sensation over the entire area of both legs. The radiographs demonstrated a L4 and L5 right transverse process fracture, first sacral vertebral fracture and symphysis pubis separation (Fig. 1). After the initial evaluation, the patient was taken to the operating room for an open reduction and internal fixation of the symphysis pubis separation with a plate and screws using the Fannenstiel approach.

After surgery and adequate medical stabilization, the patient complained of more sacral area pain as well as a progressive tingling sensation and motor weakness in both legs. The motor power on ankle plantar flexion of both legs were decreased, which was worse on the left side (2/5) than on the right (3/5). A sensory examination revealed decreased sensation in the soles bilaterally. The rectal tone and perianal sensation were decreased and the plantar reflexes were low. The postoperative computed tomography (CT) examination revealed an initially missed jumped-andlocked right L5-S1 facet joint and widened gap of the sacral fracture site resulting in cauda equine syndrome due to the stretching of the sacral roots (Fig. 2). Massive methylprednisolone was administrated intravenously but this was discontinued due to the development of a complete ileus. Two days after surgery, the patient returned to the operating room as a consequence of the progressive cauda equine syndrome that had developed despite steroid therapy and
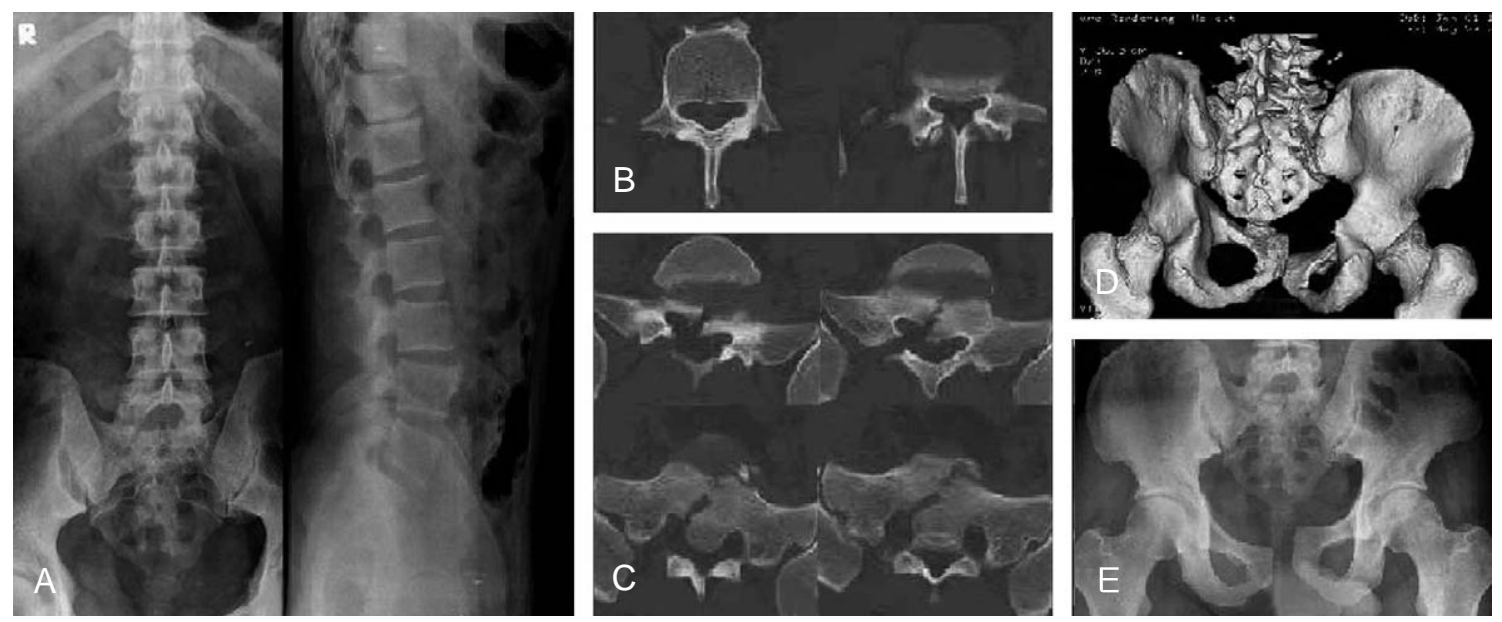

Fig. 1. Initial radiographic findings show (A) simple lumbar anterior-posterior and lateral X-ray views, (B) L4, 5 unilateral transverse process fracture on computed tomography (CT) imaging, (C) comminuted and displaced sacral fracture lines on CT imaging, (D) unilateral facet dislocation and symphysis pubis separation on three dimensional CT imaging and (E) symphysis pubis separation on initial simple pelvis anteroposterior X-ray view.

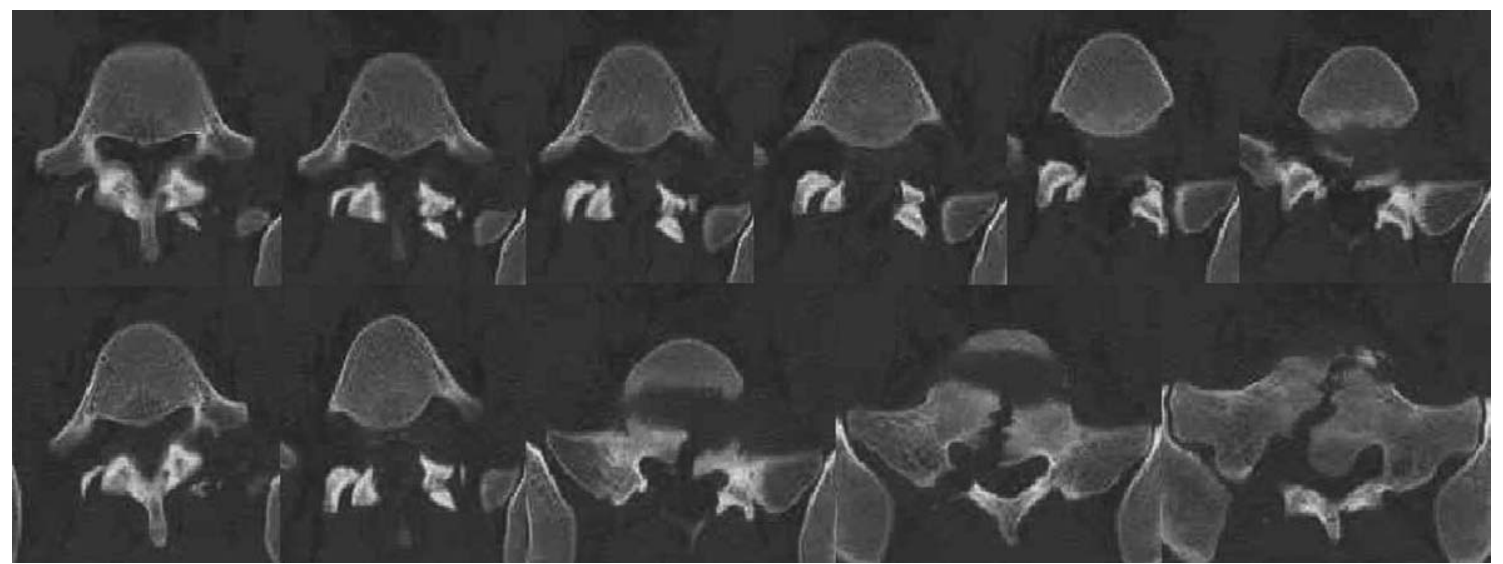

Fig. 2. Computed tomography (CT) image shows missed unilateral facet joint dislocation initially (upper row). This CT image shows a more displaced sacral fracture gap after reduction of the symphysis pubis separation (lower row). 
surgery through the posterior approach. A right L5-S1 jumped facet injury was confirmed intraoperatively without any signs of pars defects and fracture. Extensive soft tissue injury was noted with a rupture of the lumbar fascia, facet capsule and interspinous ligaments. The reduction was attempted successfully using a combination of laminar spreader distraction between the base of the L5 and S1 spinous process, towel clamp on L5 for posterior traction and a joker for the lever arm of the facet joint. After the reduction, the reduction state was confirmed by $\mathrm{C}$-arm fluoroscopy, and the post-reduction stability was confirmed during L5-S1 flexion and extension motion. A further instrumentation and fusion procedure was not considered necessary. After a second open reduction, CT demonstrated a reduced facet joint and a narrow sacral fracture gap (Fig. 3). Postoperatively, the tingling sensation decreased and there was no progressive motor weakness. Non weight bearing was permitted for 3 months due to the symphysis pubis separation. After 3 months, the patient ambulated from a wheel chair to full weight bearing. At the 6 month follow-up evaluation, the patient demonstrated marked improvement with grade $5 / 5$ and $4+/ 5$ muscle strength in the right and left plantarflexor muscles, respectively. The patient can now walk without support and the sphincter function is intact. However, he still experiences numbness in his left sole. He has since returned to his previous employment. Radiographs of the flexion/extension view revealed a normal range of movement on the L5-S1 segment.

\section{Discussion}

Unlike thoracolumbar and cervical unilateral facet dislo- cations, traumatic lumbosacral dislocations are rarely found as isolated injuries [2,3]. There are only twelve case-review reports of unilateral lumbosacral fractures dislocations (21 total cases). These lesions are produced by high-energy trauma and are often life-threatening. Ten percent of these injuries are not recognized initially due to the high mortality rate [4].

Reddy et al. [5] proposed that the injury mechanism of this lesion involves flexion-distraction forces. Vialle et al. [3] stated that the casual mechanisms were still unknown after reviewing many reports. In this case, the combination of multiple forces (compression, lateral translation, rotational force and shearing force), rather than a unidirectional force, might result in a different injury pattern. Among these forces, rotational forces should play the most important role.

The lumbosacral facet dislocation associated with symphysis pubis separation is not classified clearly with the preexisting classifications. Vialle et al. [3] suggested a new classification for a lumbosacral traumatic dislocation based on an analysis of the underlying physiopathological mechanisms of the anatomical lesions in 11 patients. Among this classification, the present case appeared to be a pure dislocation of the IA type due to a unilateral rotational dislocation secondary to a rotational traumatic force.

In this case, anteroposterior compression injury to the pelvis was added to a hyperflexion and distraction injury of the lumbosacral area, so the symphysis pubis was separated to break the sacrum and the right L5-S1 facet joint was dislocated. After reducing the separated symphysis pubis alone, the posterior sacrum fracture gap became wider and a more displaced dislocation caused further neurological symptoms with traction of the sacral nerve roots. Therefore,

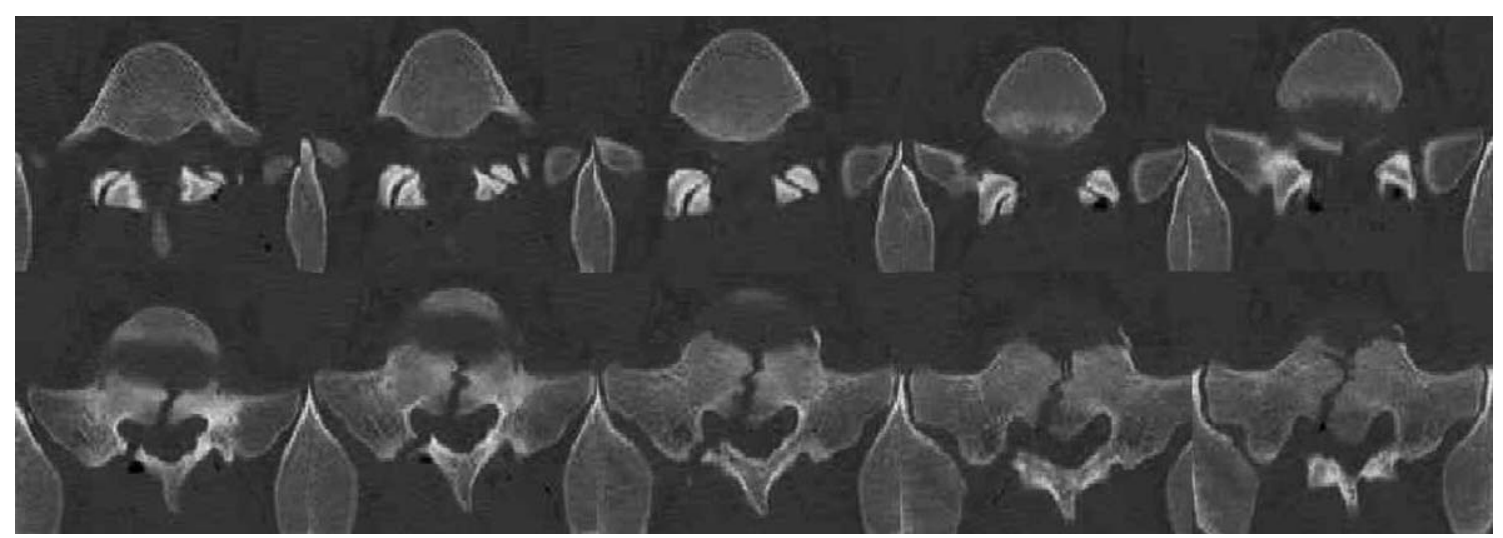

Fig. 3. This computed tomography (CT) image shows reduced L5-S1 facet joint without fusion (upper row). This CT image shows a narrowed sacral fracture gap after reduction of the L5-S1 facet joint (lower row). 
the reduction on the posterior part could result in a narrowing of the sacral gap and a decrease in the neurological deficits.

Shen et al. [4] reported that in most cases, severe neurological deficits are uncommon. De Iure et al. [6] suggested that the neurological damage to the sacral or lumbosacral roots was caused by a dual mechanism: a cutting mechanism between the anterior border of the S1 facet and the posterior wall of L5, and a stripping mechanism due to a lateral dislocation.

The treatment of a lumbosacral traumatic dislocation is controversial due to its rarity [1,3]. Almost all cases reported thus far were treated surgically with reduction and fusion with instrumentation. Veras del Monte and Bagó [7] reported a long-term follow up of patients treated non-surgically. At the 10-year follow-up, their patients showed only mild pain and maintained normal lumbar spine mobility. However, recently, the recommended treatments of this lesion were surgical reduction and fusion with instrumentation through a predominantly posterior approach [8] because the results of most studies were better with surgical intervention. A close reduction is often unsuccessful and non-surgical treatment often requires prolonged traction, which carries the risk of neurological symptoms [4]. Similarly, Shen et al. [4] suggested that an open reduction and fusion with posterior segmental instrumentation and an autologous bone graft provides the most stable construct, best change for an adequate reduction, and decreases the risk of late neurological injury secondary to the loss of reduction and fixation. Regarding the reduction, Vialle et al. [3] suggested that in cases of a neurological deficit or cauda equine syndrome, decompression surgery should be performed even in the absence of an magnetic resonance study because a close reduction of the hyperkyphotic curve of lumbosacral dislocation carries considerable risk. In this case, the facet joint was reduced easily without any forceful reduction maneuver and the reduced state was maintained stably throughout the manipulation. In addition, the left L5-S1 facet was not injured and the patient should be on bed rest for at least 2 months due to the symphysis pubis separation. The authors believed that the reduced facet joint might be well maintained throughout that period, and the possible mobility of the lumbosacral joints cannot be prevented by rigid fixation and fusion. Therefore, the surgery was completed after the reduction without rigid fixation and fusion, and a wellmaintained mobile lumbosacral segment was obtained at the last follow-up.

In conclusion, surgical treatment of an open reduction without fusion and instrumentation is a good alternative treatment for a unilateral facet dislocation without fracture. When symphysis pubis separation is diagnosed, a thorough reexamined should be performed to confirm/exclude the possibility of a posterior lumbosacral dislocation. A high index of suspicion, careful examination and appropriate radiological studies can help to obtain a correct diagnosis.

\section{REFERENCES}

1. Schmid R, Reinhold M, Blauth M. Lumbosacral dislocation: a review of the literature and current aspects of management. Injury 2010;41:321-8.

2. Szentirmai O, Seinfeld J, Beauchamp K, Patel V. Traumatic unilateral lumbosacral jumped facet without fracture in a child-presentation of a safe treatment strategy for a rare injury. Patient Saf Surg 2008;2:29.

3. Vialle R, Charosky S, Rillardon L, Levassor N, Court C. Traumatic dislocation of the lumbosacral junction diagnosis, anatomical classification and surgical strategy. Injury 2007;38:169-81.

4. Shen FH, Crowl A, Shuler TE, Feldenzer JA, Leivy SW. Delayed recognition of lumbosacral fracture dislocations in the multitrauma patient: the triad of transverse process fractures, unilateral renal contusion and lumbosacral fracture dislocation. J Trauma 2004;56:700-5.

5. Reddy SJ, Al-Holou WN, Leveque JC, La Marca F, Park P. Traumatic lateral spondylolisthesis of the lumbar spine with a unilateral locked facet: description of an unusual injury, probable mechanism, and management. J Neurosurg Spine 2008;9:576-80.

6. De Iure F, Paderni S, Gasbarrini A, Bandiera S, Boriani S. Traumatic lumbosacral lateral dislocation without fracture. Chir Organi Mov 2008;92:183-5.

7. Veras del Monte LM, Bagó J. Traumatic lumbosacral dislocation. Spine (Phila Pa 1976) 2000;25:756-9.

8. Cho SK, Lenke LG, Hanson D. Traumatic noncontiguous double fracture-dislocation of the lumbosacral spine. Spine J 2006;6:534-8. 